\title{
213.
}

\section{ON THE DEVELOPMENT OF THE DISTURBING FUNCTION IN THE LUNAR THEORY.}

[From the Memoirs of the Royal Astronomical Society, vol. xxvII., (1859), pp. 69-95. Read November 12, 1858.]

THE development of the disturbing function for the lunar theory is effected in a very elegant manner in Hansen's Fundanienta Nova, and it requires only a single easy step to exhibit the result in a perfectly explicit form, and to compare it with those of other geometers. To do this is the immediate object of the present memoir, and the mode of development is a mere reproduction of that made use of by Hansen. But the memoir is written with a view to the development of and application to the lunar theory, of the theory contained in my "Memoir on the Problem of Disturbed Elliptic Motion," ante pp. 1-29, [212], and the notation adopted (differing from Hansen's very slightly) is consequently that of the memoir just referred to.

Taking, as usual, $\Omega$ to denote the disturbing function with the sign employed by Lagrange ( $\Omega=-R$, if $R$ be the disturbing function of the Mécanique Céleste), then

$$
\Omega=m^{\prime}\left\{\frac{1}{\left(r^{2}+r^{\prime 2}-2 r r^{\prime} \cos H\right)^{\frac{1}{2}}}-\frac{r}{r^{\prime 2}} \cos H\right\},
$$

where we have

$$
\begin{aligned}
& m^{\prime} \text {, the mass of the sun, } \\
& r \text {, the radius vector of the moon, } \\
& r^{\prime} \text {, the radius vector of the sun, } \\
& H \text {, the angular distance of the sun and moon, }
\end{aligned}
$$

the earth being, of course, taken as the centre of motion; (Hansen's $\Omega$ is the above value divided by $M+m$, where $M$ and $m$ are the masses of the earth and moon respectively; that is, the disturbing function here represented by $\Omega$ is Hansen's $\Omega$ multiplied into $M+m$ ). 
Write also
$f$, the true anomaly of the moon,
$f^{\prime}$, the true anomaly of the sun,
$\boldsymbol{\nearrow}$, the distance of moon's pericentre from ascending node of moon's orbit,
$\boldsymbol{\tau}^{\prime}$, the distance of sun's pericentre from same node,
$\Phi$, the inclination of moon's orbit to that of the sun,

where the orbits referred to are the true or instantaneous orbits. Then the angular distance $H$ is the third side of a spherical triangle, the other two sides whereof are $f+\boldsymbol{\tau}, f^{\prime}+\boldsymbol{\mho}^{\prime}$, the included angle between them being $\Phi$, that is, we have

$$
\cos H=\cos (f+\boldsymbol{\tau}) \cos \left(f^{\prime}+\boldsymbol{乙}^{\prime}\right)+\cos \Phi \sin (f+\boldsymbol{\tau}) \sin \left(f^{\prime}+\boldsymbol{乙}^{\prime}\right)
$$

and in this equation, if we write

$$
\begin{aligned}
& g \text {, the mean anomaly of the moon, } \\
& a \text {, the semi-axis major of the moon's orbit, } \\
& e, \text { the eccentricity; }
\end{aligned}
$$

and in like manner,

$g^{\prime}$, the mean anomaly of the sun,

$a^{\prime}$, the semi-axis major of the sun's orbit,

$\epsilon^{\prime}$, the eccentricity;

then $r, f$, and $r^{\prime}, f^{\prime}$, are respectively given functions of $a, e, g$, and $a^{\prime}, e^{\prime}, g^{\prime}$, viz., we have

$$
\begin{aligned}
& r=a \text { elqr }(e, g), \\
& f=\text { elta }(e, g), \\
& r^{\prime}=a^{\prime} \text { elqr }\left(e^{\prime}, g^{\prime}\right) \\
& f^{\prime}=\operatorname{elta}\left(e^{\prime}, g^{\prime}\right)
\end{aligned}
$$

and introducing, instead of the inclination, the quantity

$$
\eta\left(=\sin \frac{1}{2} \Phi\right), \text { the sine of the semi-inclination; }
$$

the disturbing function $\Omega$ becomes a function of $a, e, g, \boldsymbol{\tau}, a^{\prime}, e^{\prime}, g^{\prime}, \boldsymbol{\tau}^{\prime}, \eta$, and the required development is a development in multiple cosines of $g, g^{\prime}, \boldsymbol{\tau}, \boldsymbol{\tau}^{\prime}$, the coefficients being of course functions of the remaining quantities $a, e, a^{\prime}, e^{\prime}, \eta$. The single symbols $\boldsymbol{\tau}, \boldsymbol{\tau}^{\prime}$ (which denote the distances of the pericentres of the lunar and solar orbits from the mutual node) will be retained throughout the memoir; but if we write

$$
\begin{aligned}
& \varpi, \text { the departure of moon's pericentre, } \\
& \Sigma \text {, the departure of moon's ascending node; }
\end{aligned}
$$


these departures being measured on the moon's orbit; and, in like manner, measured on the sun's orbit from a departure point on this orbit, but called for distinction "longitudes," instead of departures,

$$
\begin{aligned}
& \varpi^{\prime} \text {, the longitude of the sun's pericentre, } \\
& \Theta \text {, the longitude of the moon's ascending node; }
\end{aligned}
$$

then we have

$$
\begin{aligned}
& \tau=\varpi-\Sigma, \\
& \tau^{\prime}=\varpi^{\prime}-\Theta ;
\end{aligned}
$$

and the disturbing function $\Omega$, so far as it depends on the position of the moon, is a function of the seven elements $a, e, g, \varpi, \eta, \Sigma, \Theta$, and it contains also the quantities $a^{\prime}, e^{\prime}, g^{\prime}, \varpi^{\prime}$, which relate to the sun.

Proceeding now to develope $\Omega$ in ascending powers of $\frac{r}{r^{\prime}}$, we have

$$
\Omega=m^{\prime}\left\{\frac{1}{r^{\prime}}+\frac{r^{2}}{r^{\prime 3}}\left[\frac{3}{2} \cos ^{2} H-\frac{1}{2}\right]+\frac{r^{3}}{r^{\prime 4}}\left[\frac{5}{2} \cos ^{3} H-\frac{3}{2} \cos H\right]+\frac{r^{4}}{r^{\prime 5}}\left[\frac{35}{8} \cos ^{4} H-\frac{15}{4} \cos ^{2} H+\frac{3}{8}\right]+\& c .\right\}
$$

where, however, the last term is neglected in the sequel, and since we are only concerned with the differential coefficients of $\Omega$ in regard to the lunar elements, the first term $m^{\prime} \frac{1}{r^{\prime}}$ which depends only on the solar elements may also be neglected; we have

$$
\begin{aligned}
& \cos H=\cos ^{2} \frac{1}{2} \Phi \quad \cos \left(f-f^{\prime}+\tau-\tau^{\prime}\right) \\
& +\sin ^{2} \frac{1}{2} \Phi \quad \cos \left(f+f^{\prime}+\tau+\tau^{\prime}\right),
\end{aligned}
$$

and thence

$$
\begin{aligned}
& \cos ^{2} H=\cos ^{4} \frac{1}{2} \Phi \quad \cos ^{2}\left(f-f^{\prime}+\tau-\tau^{\prime}\right) \\
& +2 \cos ^{2} \frac{1}{2} \Phi \sin ^{2} \frac{1}{2} \Phi \cos \left(f-f^{\prime}+\tau-\tau^{\prime}\right) \cos \left(f+f^{\prime}+\tau+\tau^{\prime}\right) \\
& +\sin ^{4} \frac{1}{2} \Phi \quad \cos ^{2}\left(f+f^{\prime}+\tau+\tau^{\prime}\right) \text {, } \\
& \cos ^{3} H=\cos ^{6} \frac{1}{2} \Phi \quad \cos ^{3}\left(f-f^{\prime}+\boldsymbol{\tau}-\boldsymbol{乙}^{\prime}\right) \\
& +3 \cos ^{4} \frac{1}{2} \Phi \sin ^{2} \frac{1}{2} \Phi \cos ^{2}\left(f-f^{\prime}+\tau-\tau^{\prime}\right) \cos \left(f+f^{\prime}+\tau+\tau^{\prime}\right) \\
& +3 \cos ^{2} \frac{1}{2} \Phi \sin ^{4} \frac{1}{2} \Phi \cos \left(f-f^{\prime}+\tau-\tau^{\prime}\right) \cos ^{2}\left(f+f^{\prime}+\tau+\boldsymbol{乙}^{\prime}\right) \\
& +\sin ^{6} \frac{1}{2} \Phi \quad \cos ^{3}\left(f+f^{\prime}+\tau+\tau^{\prime}\right) \text {, }
\end{aligned}
$$

and converting the powers of the cosines of $f-f^{\prime}+\boldsymbol{\tau}-\boldsymbol{\mho}^{\prime}, f+f^{\prime}+\boldsymbol{\tau}+\boldsymbol{\mho}^{\prime}$ into multiple cosines, and expressing the coefficients in terms of $\eta\left(=\sin \frac{1}{2} \Phi\right)$ and neglecting $\eta^{6}$, we have 


$$
\begin{aligned}
& \cos H=-\eta^{2} \quad \cos f-f^{\prime}+\tau-\boldsymbol{\tau}^{\prime} \\
& +\eta^{2} \quad \cos f+f^{\prime}+\tau+\boldsymbol{\tau}^{\prime}, \\
& \cos ^{2} H=\frac{1}{2}-\eta^{2}+\eta^{4} \\
& +\frac{1}{2}-\eta^{2}+\frac{1}{2} \eta^{4} \quad \cos 2 f-2 f^{\prime}+2 \mho-2 \mho^{\prime} \\
& +\eta^{2}-\eta^{4} \cos 2 f+2 \tau \\
& +\eta^{2}-\eta^{4} \cos 2 f^{\prime}+2 \boldsymbol{U}^{\prime} \\
& +\quad \frac{1}{2} \eta^{4} \quad \cos 2 f+2 f^{\prime}+2 \boldsymbol{\tau}+2 \boldsymbol{乙}^{\prime}, \\
& \cos ^{3} H=\frac{3}{4}-\frac{9}{4} \eta^{2}+\frac{15}{4} \eta^{4} \quad \cos f-f^{\prime}+\boldsymbol{\tau}-\boldsymbol{\mho}^{\prime} \\
& +\frac{1}{4}-\frac{3}{4} \eta^{2}+\frac{3}{4} \eta^{4} \quad \cos 3 f-3 f^{\prime}+3 \tau-3 \boldsymbol{\mho}^{\prime} \\
& +\frac{3}{2} \eta^{2}-3 \eta^{4} \quad \cos f+f^{\prime}+\boldsymbol{\tau}+\boldsymbol{\mho}^{\prime} \\
& +\frac{3}{4} \eta^{2}-\frac{3}{2} \eta^{4} \quad \cos 3 f-f^{\prime}+3 \boldsymbol{\tau}-\boldsymbol{\mho}^{\prime} \\
& +\quad \frac{3}{4} \eta^{2}-\frac{3}{2} \eta^{4} \quad \cos f-3 f^{\prime}+\nearrow-3 \boldsymbol{\mho}^{\prime} \\
& +\quad \frac{3}{4} \eta^{4} \quad \cos 3 f+f^{\prime}+3 \boldsymbol{\zeta}+\boldsymbol{\tau}^{\prime} \\
& +\quad \frac{3}{4} \eta^{4} \cos f+3 f^{\prime}+\mho+3 \boldsymbol{\mho}^{\prime} \text {. }
\end{aligned}
$$

Hence

$\Omega=m^{\prime} \frac{r^{2}}{r^{\prime 3}}$ multiplied into

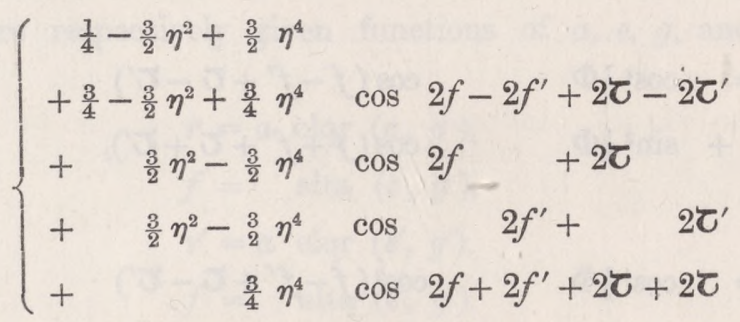

$+m^{\prime} \frac{r^{3}}{r^{\prime 4}}$ multiplied into

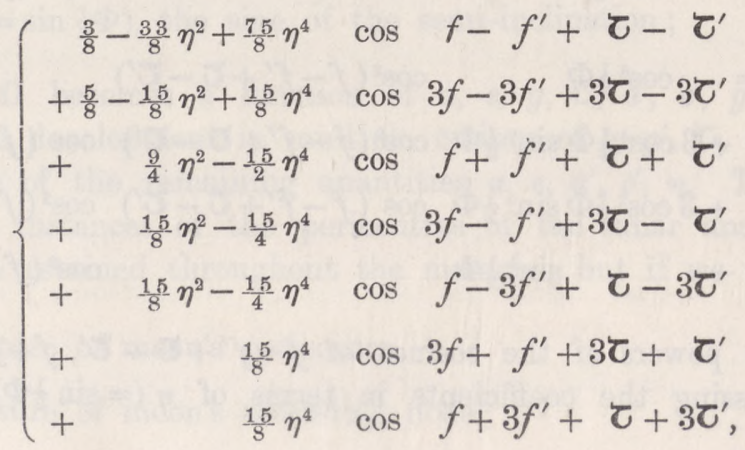


but the last two terms of the part multiplied by $m^{\prime} \frac{r^{3}}{r^{\prime 4}}$ (which are besides of the fourth order in $\eta$ ) are neglected in the sequel.

Now $i$, $i^{\prime}$ denoting integer numbers, and writing down only the general terms (the summatory sign $\Sigma_{-\infty}^{\infty}$ being in each case understood), we may put

$$
\begin{aligned}
& \frac{r^{2}}{a^{2}} \cos 2 f=Q_{c}^{i} \cos i g, \frac{r^{2}}{a^{2}} \sin 2 f=Q_{s}{ }^{i} \sin i g, \\
& \frac{r^{2}}{a^{2}} \quad=P^{i} \cos i g, \\
& \frac{a^{\prime 3}}{r^{\prime 3}} \cos 2 f^{\prime}=G_{c}^{i} \cos i^{\prime} g^{\prime}, \quad \frac{a^{\prime 3}}{r^{\prime 3}} \sin 2 f^{\prime}=G_{s}^{i^{\prime}} \sin i^{\prime} g^{\prime}, \\
& \frac{a^{\prime 3}}{r^{\prime 3}} \quad=K^{i^{\prime}} \cos i^{\prime} g^{\prime}, \\
& \frac{r^{3}}{a^{3}} \cos f=A_{c}{ }^{i} \cos i g, \quad \frac{r^{3}}{a^{3}} \sin f=A_{s}^{i} \sin i g, \\
& \frac{r^{3}}{a^{3}} \cos 3 f=B_{c}^{i} \cos i g, \quad \frac{r^{3}}{a^{3}} \sin 3 f=B_{s}{ }^{i} \sin i g, \\
& \frac{a^{\prime 4}}{r^{\prime 4}} \cos f^{\prime}=C_{c}^{i^{\prime}} \cos i^{\prime} g^{\prime}, \quad \frac{a^{\prime 4}}{r^{\prime 4}} \sin f^{\prime}=C_{s}^{i^{\prime}} \sin i^{\prime} g^{\prime}, \\
& \frac{a^{\prime 4}}{r^{\prime 4}} \cos 3 f^{\prime}=D_{c}^{i^{\prime}} \cos i^{\prime} g^{\prime}, \quad \frac{a^{\prime 4}}{r^{\prime 4}} \sin 3 f^{\prime}=D_{s}^{i^{\prime \prime}} \sin i^{\prime} g^{\prime},
\end{aligned}
$$

where

$$
\begin{aligned}
& Q_{c}^{i}=Q_{c}^{-i}, \quad G_{c}^{i^{\prime}}=G_{c}^{-i^{\prime}}, \quad A_{c}^{i}=A_{e}^{-i}, \& c . \\
& Q_{s}^{i}=-Q_{s}{ }^{-i}, \quad G_{s}^{i^{\prime}}=-G_{s}^{-i^{\prime}}, \quad A_{s}^{i}=-A_{s}^{-i}, \& c . \\
& P^{i}=P^{-i}, \quad K^{i^{\prime}}=K^{-i^{\prime}} .
\end{aligned}
$$

Then by a known rule for the multiplication of doubly infinite sine or cosine series, and after an easy transformation of the original form of the coefficients, we obtain, for instance,

$$
\frac{r^{2}}{r^{\prime 3}} \cos \left(2 f-2 f^{\prime}+2 \boldsymbol{\tau}-2 \boldsymbol{\mho}^{\prime}\right)=\frac{a^{2}}{a^{\prime 3}}\left(Q_{c}{ }^{i}+Q_{s}{ }^{i}\right)\left(G_{c}{ }^{i^{\prime}}-G_{s}^{i^{\prime}}\right) \cos \left(i g+i^{\prime} g^{\prime}+2 \boldsymbol{\tau}-2 \boldsymbol{乙}^{\prime}\right)
$$

where only the general term is written down, but the indices $i$, $i^{\prime}$, each of them extend from $-\infty$ to $+\infty$, zero included, or the summatory sign $\Sigma_{-\infty}^{\infty} \Sigma_{-\infty}^{\infty}$ is to be understood; and similarly for the other terms of $\Omega$. And we may write

$$
\Omega=\Omega_{1}+\Omega_{2}+\Omega_{3}+\Omega_{4}+\Omega_{5}+\Omega_{6}+\Omega_{7}+\Omega_{8}+\Omega_{9}+\Omega_{10},
$$

C. III. 
where

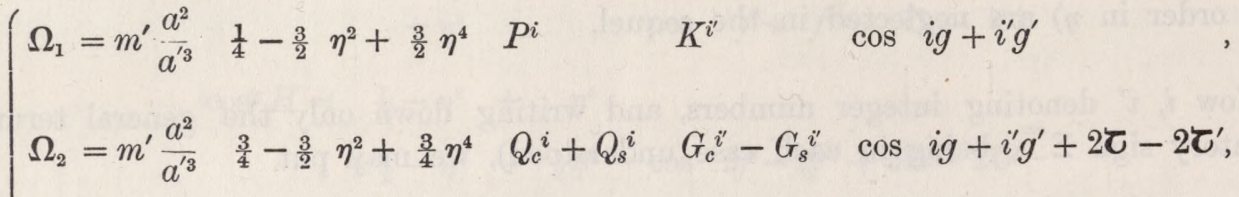

$$
\begin{aligned}
& \Omega_{3}=m^{\prime} \frac{a^{2}}{a^{\prime 3}} \quad \frac{3}{2} \eta^{2}-\frac{3}{2} \eta^{4} \quad Q_{c}{ }^{i}+Q_{s}{ }^{i} \quad K^{i^{\prime}} \quad \cos \quad i g+i^{\prime} g^{\prime}+2 \tau \\
& \Omega_{4}=m^{\prime} \frac{a^{2}}{a^{\prime 3}} \quad \frac{3}{2} \eta^{2}-\frac{3}{2} \eta^{4} \quad P^{i} \quad G_{c}^{i^{\prime}}+G_{s}^{i^{\prime}} \quad \cos i g+i^{\prime} g^{\prime} \quad+2 \boldsymbol{\mho}^{\prime} \\
& \Omega_{5}=m^{\prime} \frac{a^{2}}{a^{\prime 3}} \quad \frac{3}{4} \eta^{4} \quad Q_{c}{ }^{i}+Q_{s}{ }^{i} \quad G_{c}^{i^{\prime}}+G_{s}^{i^{\prime}} \quad \cos \quad i g+i^{\prime} g^{\prime}+2 \boldsymbol{\tau}+2 \boldsymbol{乙}^{\prime} \\
& \left(\Omega_{6}=m^{\prime} \frac{a^{3}}{a^{\prime 4}} \frac{3}{8}-\frac{33}{8} \eta^{2}+\frac{75}{8} \eta^{4} \quad A_{c}^{i}+A_{s}^{i} \quad C_{c}^{i^{\prime}}-C_{s}^{i^{\prime}} \quad \cos i g+i^{\prime} g^{\prime}+\tau-\boldsymbol{\nearrow}^{\prime},\right. \\
& \Omega_{7}=m^{\prime} \frac{a^{3}}{a^{\prime 4}} \frac{5}{8}-\frac{15}{8} \eta^{2}+\frac{15}{8} \eta^{4} \quad B_{c}{ }^{i}+B_{s}{ }^{i} \quad D_{c}^{i^{\prime}}-D_{s}^{i^{\prime}} \quad \cos \quad i g+i^{\prime} g^{\prime}+3 \circlearrowright-3 \boldsymbol{乙}^{\prime}, \\
& \Omega_{8}=m^{\prime} \frac{a^{3}}{a^{\prime 4}} \quad \frac{9}{4} \eta^{2}-\frac{15}{2} \eta^{4} \quad A_{c}^{i}+A_{s}^{i} \quad C_{c}^{i^{\prime}}+C_{8}^{i^{\prime}} \quad \cos i g+i^{\prime} g^{\prime}+乙+\boldsymbol{\circlearrowright}^{\prime}, \\
& \Omega_{9}=m^{\prime} \frac{a^{3}}{a^{\prime 4}} \quad \frac{15}{8} \eta^{2}-\frac{15}{4} \eta^{4} \quad B_{c}{ }^{i}+B_{s}{ }^{i} \quad C_{c}^{i^{\prime}}-C_{s}^{i^{\prime}} \quad \cos i g+i^{\prime} g^{\prime}+3 \circlearrowright-\boldsymbol{\circlearrowright}^{\prime}, \\
& \Omega_{10}=m^{\prime} \frac{a^{3}}{a^{\prime 4}} \quad \frac{15}{8} \eta^{2}-\frac{15}{4} \eta^{4} \quad A_{c}^{i}+A_{s}^{i} \quad D_{c}^{i^{\prime}}-D_{s}^{i^{\prime}} \quad \cos \quad i g+i^{\prime} g^{\prime}+\text { て } 3 \boldsymbol{\mho}^{\prime} .
\end{aligned}
$$

The values of $P^{i}, Q_{c}{ }^{i}, Q_{s}{ }^{i}$, \&c. are made to depend ultimately upon the development in multiple cosines of the square of the radius vector and the development in multiple sines of the true anomaly. And the actual values of $P^{i}, Q_{c}{ }^{i}, Q_{s}{ }^{i}, \& c$. , expanded in powers of $e$ or $e^{\prime}$, are given pp. $174-179$ of the work above referred to. I have verified all these values by a different process, and have discovered only a single inaccuracy, which, however, is rather an important one, as it affects the evection, viz., the value of $Q_{s}{ }^{1}$ should be $-\frac{3}{2} e+\frac{23}{24} e^{3}+\& c$. instead of (Hansen) $-\frac{3}{2} e+\frac{33}{24} e^{3}+\& c$. The formation of the sums or differences $Q_{c}{ }^{i}+Q_{s}{ }^{i}$ is of course perfectly easy, and we thus obtain the actual developed expression of the disturbing function, which I represent under the following form, viz.:-

$$
\Omega=\Omega_{1}+\Omega_{2}+\Omega_{3}+\Omega_{4}+\Omega_{5}+\Omega_{6}+\Omega_{7}+\Omega_{8}+\Omega_{9}+\Omega_{10},
$$

where 
$\Omega_{1}=m^{\prime} \frac{a^{2}}{a^{\prime 3}}\left(\frac{1}{4}-\frac{3}{2} \eta^{2}+\frac{3}{2} \eta^{4}\right)$ multiplied into

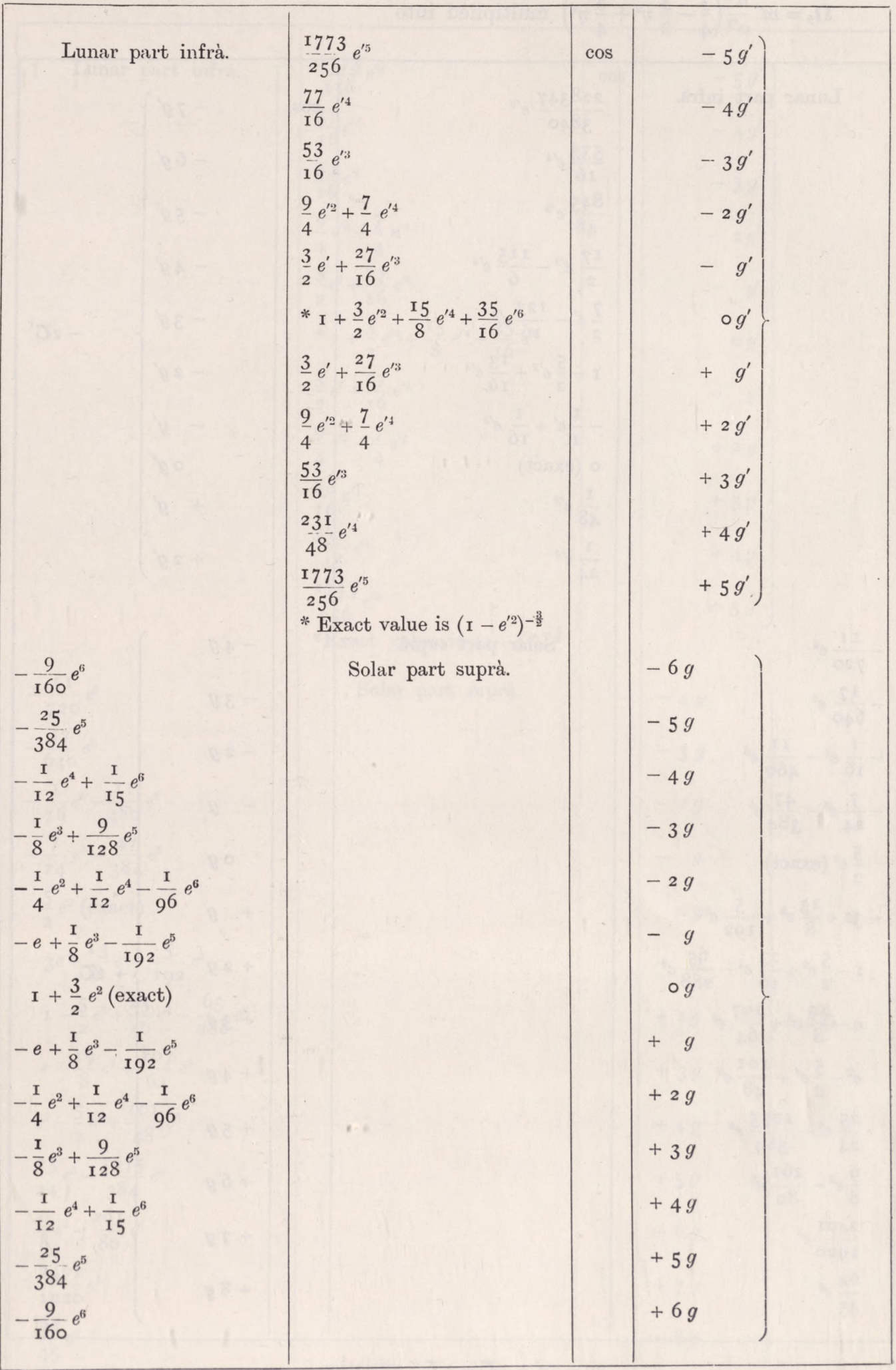


$\Omega_{2}=m^{\prime} \frac{a^{2}}{a^{\prime 3}}\left(\frac{3}{4}-\frac{3}{2} \eta^{2}+\frac{3}{4} \eta^{4}\right)$ multiplied into

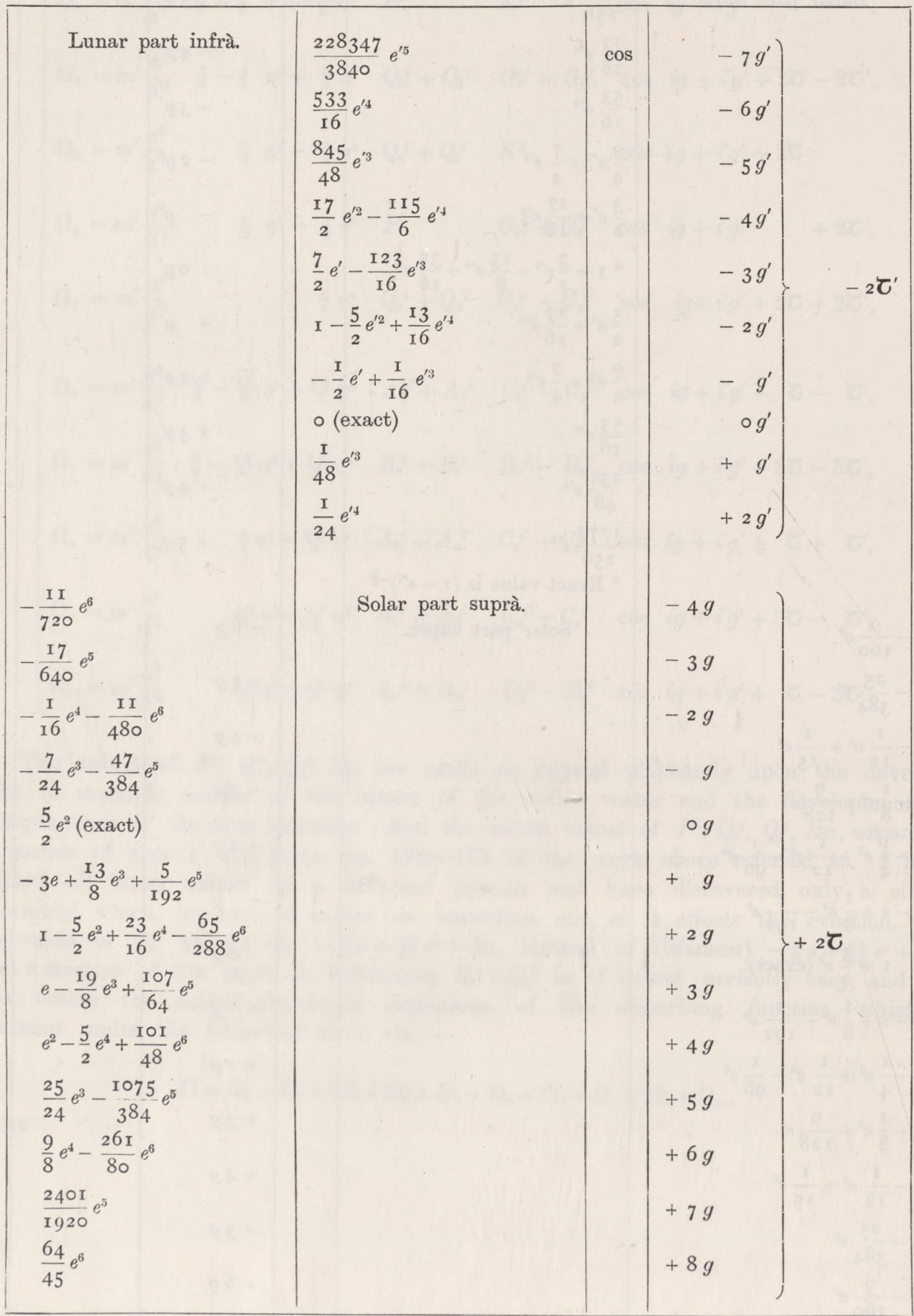

$2 g-2 g^{\prime}+2 \tau-2 \boldsymbol{U}^{\prime}$ variation. 
$\Omega_{3}=m^{\prime} \frac{a^{2}}{a^{\prime 3}}\left(\frac{3}{2} \eta^{2}-\frac{3}{2} \eta^{4}\right)$ multiplied into

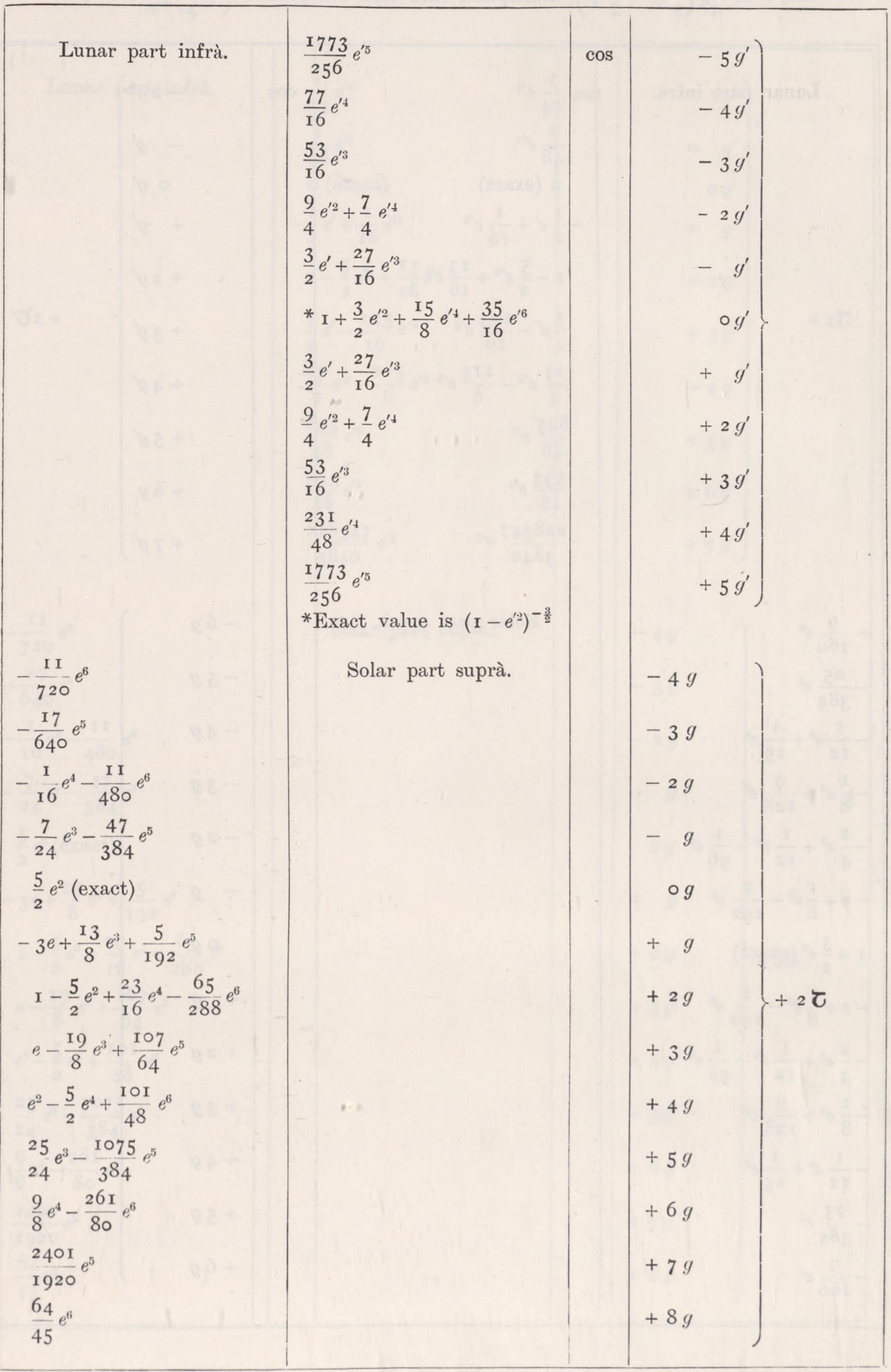


$\Omega_{4}=m^{\prime} \frac{a^{2}}{a^{\prime 3}}\left(\frac{3}{2} \eta^{2}-\frac{3}{2} \eta^{4}\right)$ multiplied into

\begin{tabular}{|c|c|c|c|c|}
\hline $\begin{array}{l}-\frac{9}{160} e^{6} \\
-\frac{25}{384} e^{5} \\
-\frac{\mathrm{I}}{\mathrm{I} 2} e^{4}+\frac{\mathrm{I}}{\mathrm{I} 5} e^{6} \\
-\frac{\mathrm{I}}{8} e^{3}+\frac{9}{\mathrm{I} 28} e^{5} \\
-\frac{\mathrm{I}}{4} e^{2}+\frac{\mathrm{I}}{\mathrm{I} 2} e^{4}-\frac{\mathrm{I}}{96} e^{6} \\
-e+\frac{\mathrm{I}}{8} e^{3}-\frac{\mathrm{I}}{\mathrm{I} 9^{2}} e^{5} \\
\mathrm{I}+\frac{3}{2} e^{2}(\mathrm{exact}) \\
-e+\frac{\mathrm{I}}{8} e^{3}-\frac{\mathrm{I}}{\mathrm{I} 9^{2}} e^{5} \\
-\frac{\mathrm{I}}{4} e^{2}+\frac{\mathrm{I}}{\mathrm{I} 2} e^{4}-\frac{\mathrm{I}}{96} e^{6} \\
-\frac{\mathrm{I}}{8} e^{3}+\frac{9}{\mathrm{I} 28} e^{5} \\
-\frac{\mathrm{I}}{\mathrm{I} 2} e^{4}+\frac{\mathrm{I}}{\mathrm{I} 5} e^{6} \\
-\frac{25}{384} e^{5} \\
-\frac{9}{\mathrm{I} 60} e^{6}\end{array}$ & $\begin{array}{l}\frac{1}{24} e^{\prime 4} \\
\frac{1}{48} e^{\prime 3} \\
\circ(\text { exact }) \\
-\frac{1}{2} e^{\prime}+\frac{1}{16} e^{\prime 3} \\
1-\frac{5}{2} e^{\prime 2}+\frac{13}{16} e^{t^{4}} \\
\frac{7}{2} e^{\prime}-\frac{123}{16} e^{\prime 3} \\
\frac{17}{2} e^{\prime 2}-\frac{115}{6} e^{\prime 4} \\
\frac{845}{48} e^{\prime 3} \\
\frac{533}{16} e^{\prime 4} \\
\frac{228347}{3840} e^{\prime 5} \\
\quad \text { Solar part suprà. }\end{array}$ & $\cos$ & $\begin{array}{l}-2 g^{\prime} \\
-\quad g^{\prime} \\
\circ g^{\prime} \\
+\quad g^{\prime} \\
+2 g^{\prime} \\
+3 g^{\prime} \\
+4 g^{\prime} \\
+5 g^{\prime} \\
+6 g \\
+7 g^{\prime}\end{array}$ & $+2 \boldsymbol{U}^{\prime}$ \\
\hline
\end{tabular}


$\Omega_{5}=m^{\prime} \frac{a^{9}}{a^{\prime 3}}\left(\frac{3}{4} \eta^{4}\right)$ multiplied into

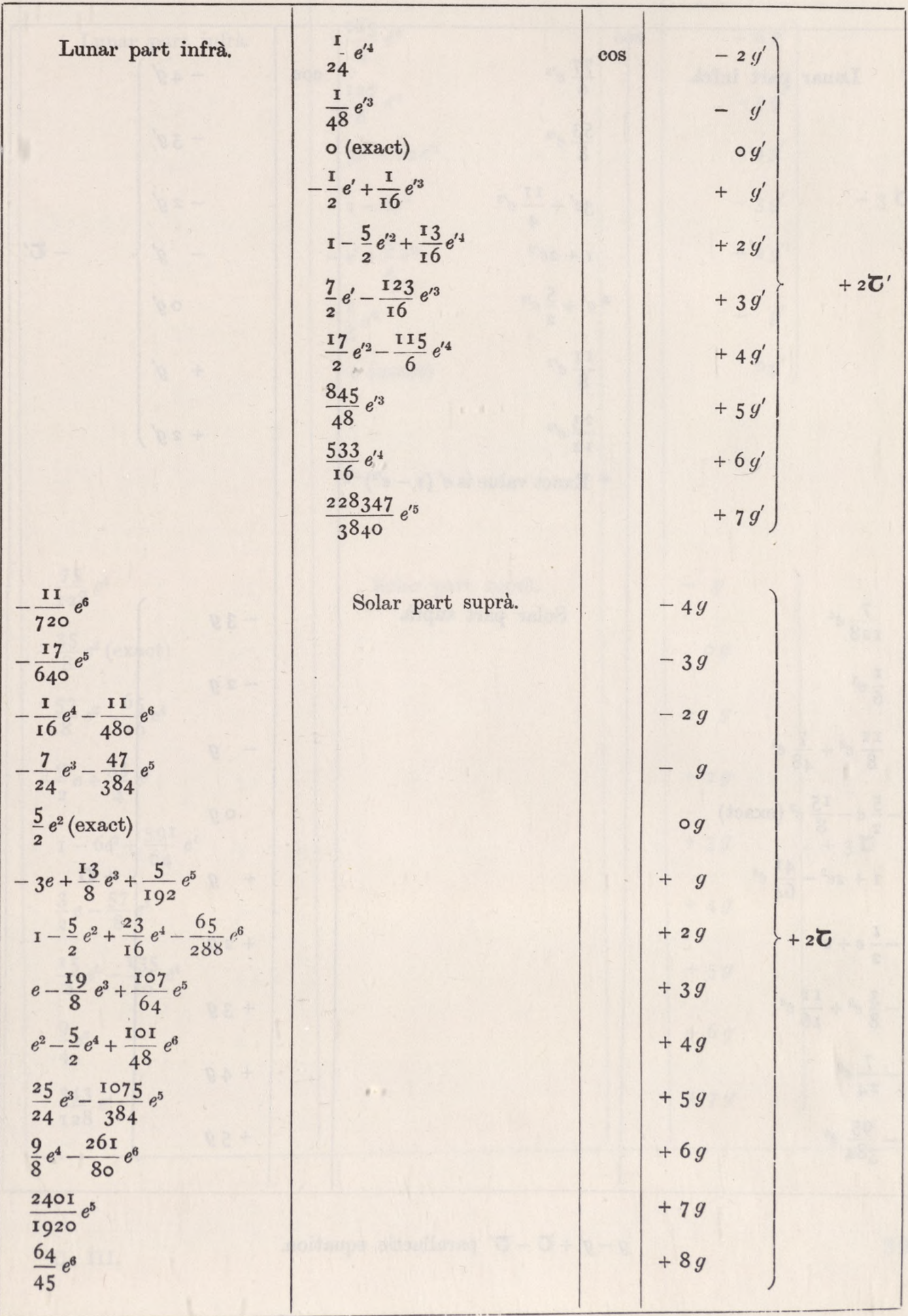


$\Omega_{6}=m^{\prime} \frac{a^{3}}{a^{4}}\left(\frac{3}{8}-\frac{33}{8} \eta^{2}+\frac{75}{8} \eta^{4}\right)$ multiplied into

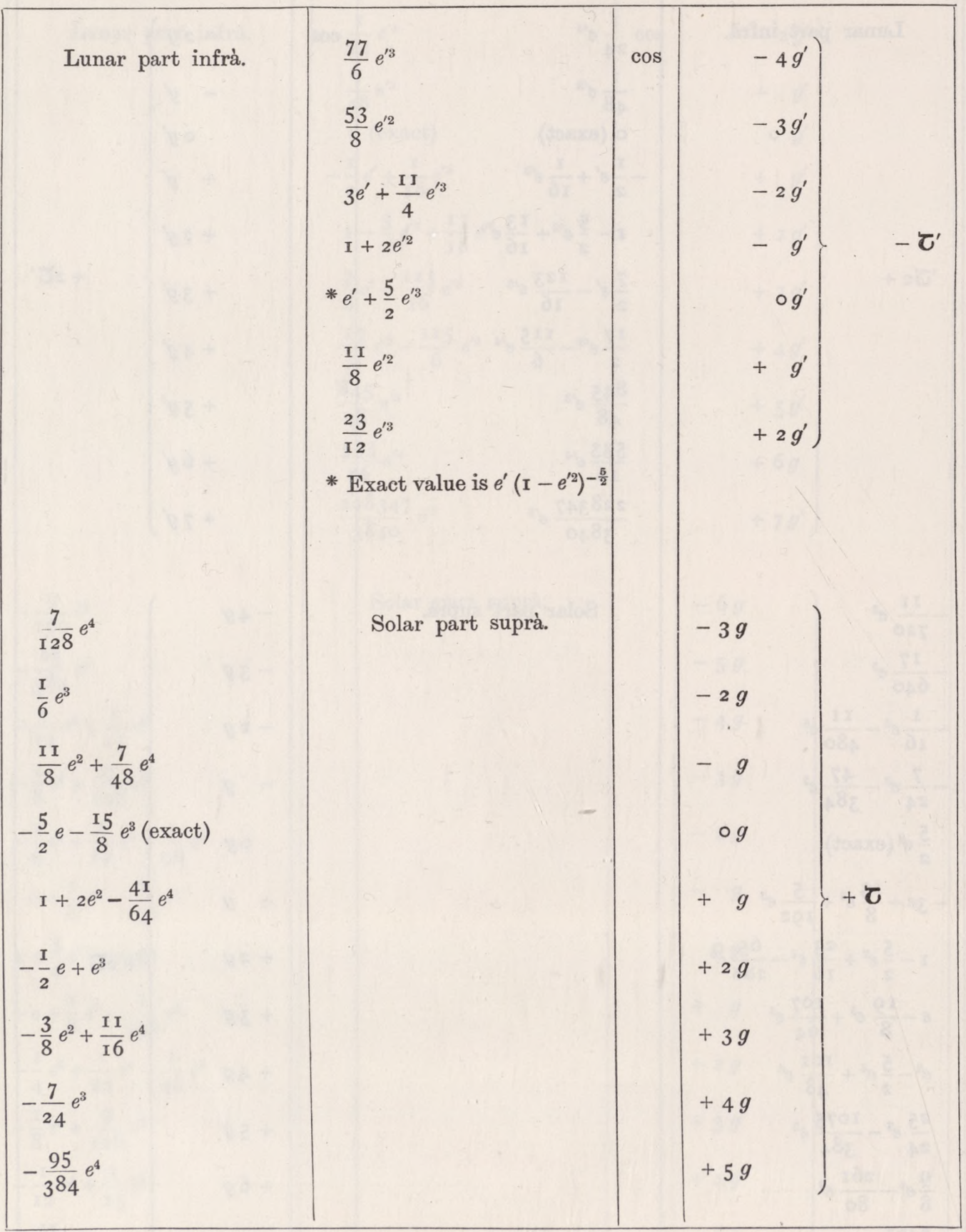


$\Omega_{7}=m^{\prime} \frac{a^{3}}{a^{44}}\left(\frac{5}{8}-\frac{15}{8} \eta^{2}+\frac{15}{8} \eta^{4}\right)$ multiplied into

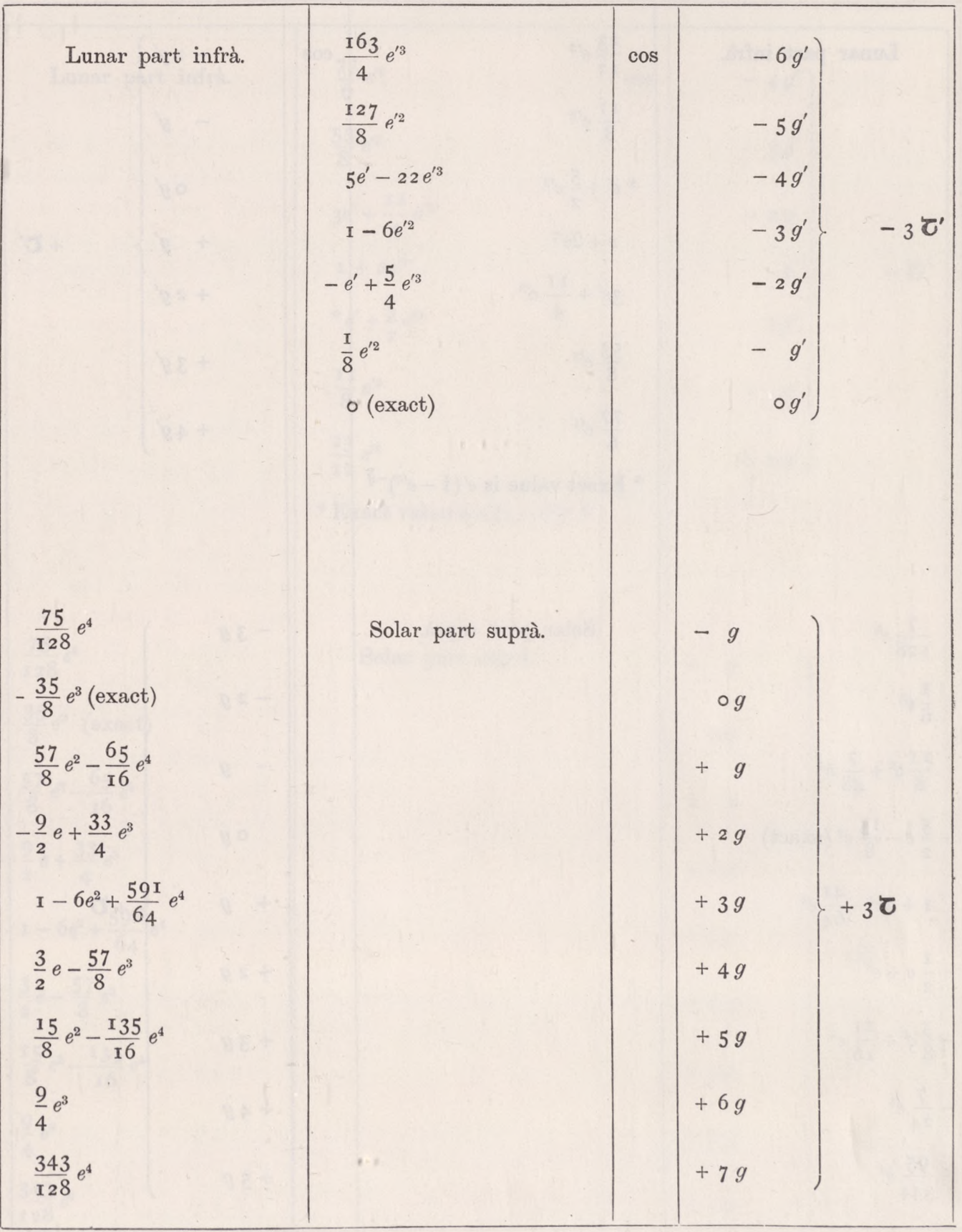

C. III. 
$\Omega_{8}=m^{\prime} \frac{a^{3}}{a^{j_{4}}}\left(\frac{9}{4} \eta^{2}-\frac{15}{2} \eta^{4}\right)$ multiplied into

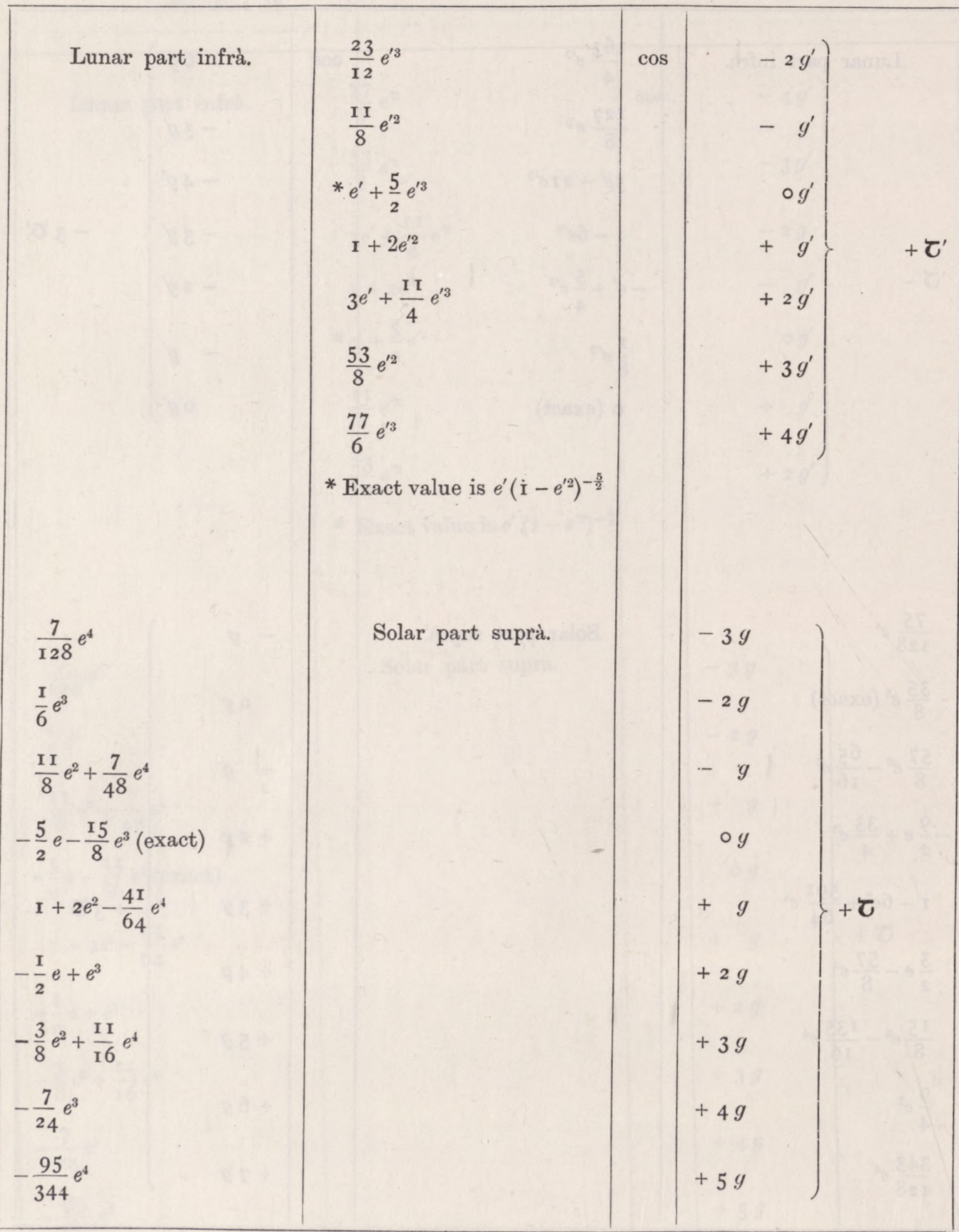


$\Omega_{9}=m^{\prime} \frac{a^{3}}{a^{\prime 4}}\left(\frac{15}{8} \eta^{2}-\frac{15}{4} \eta^{4}\right)$ multiplied into

\begin{tabular}{|c|c|c|c|c|}
\hline Lunar part infrà. & $\begin{array}{l}\frac{77}{6} e^{\prime 3} \\
\frac{53}{8} e^{\prime 2} \\
3 e^{\prime}+\frac{I I}{4} e^{\prime 3} \\
\mathrm{I}+2 e^{\prime 2} \\
* e^{\prime}+\frac{5}{2} e^{\prime 3} \\
\frac{\mathrm{II}}{8} e^{\prime 2} \\
\frac{23}{\mathrm{I} 2} e^{\prime 3} \\
\text { * Exact value is } e^{\prime}\left(\mathrm{I}-e^{\prime 2}\right)^{-\frac{5}{2}}\end{array}$ & $\cos$ & $\begin{array}{r}-4 g^{\prime} \\
-3 g^{\prime} \\
-2 g^{\prime} \\
-\quad g^{\prime} \\
\circ g^{\prime} \\
+\quad g^{\prime} \\
+2 g^{\prime}\end{array}$ & $-\boldsymbol{\nearrow}^{\prime}$ \\
\hline$\frac{75}{128} e^{4}$ & Solar part suprà. & & $-g$ & \\
\hline$-\frac{35}{8} e^{3} \quad$ (exact) & & & & \\
\hline$\frac{57}{8} e^{2}-\frac{65}{16} e^{4}$ & & & & \\
\hline$-\frac{9}{2} e+\frac{33}{4} e^{3}$ & & & $+2 g$ & \\
\hline$I-6 e^{2}+\frac{59 I}{64} e^{4}$ & & & $+3 g$ & $+3 \tau$ \\
\hline$\frac{3}{2} e-\frac{57}{8} e^{3}$ & & & & \\
\hline$\frac{15}{8} e^{2}-\frac{135}{16} e^{4}$ & & & & \\
\hline$\frac{9}{4} e^{3}$ & A. & & $+6 g$ & \\
\hline$\frac{343}{128} e^{4}$ & & & & \\
\hline
\end{tabular}


$\Omega_{10}=m^{\prime} \frac{a^{3}}{a^{a_{4}}}\left(\frac{15}{8} \eta^{2}-\frac{15}{4} \eta^{4}\right)$ multiplied into

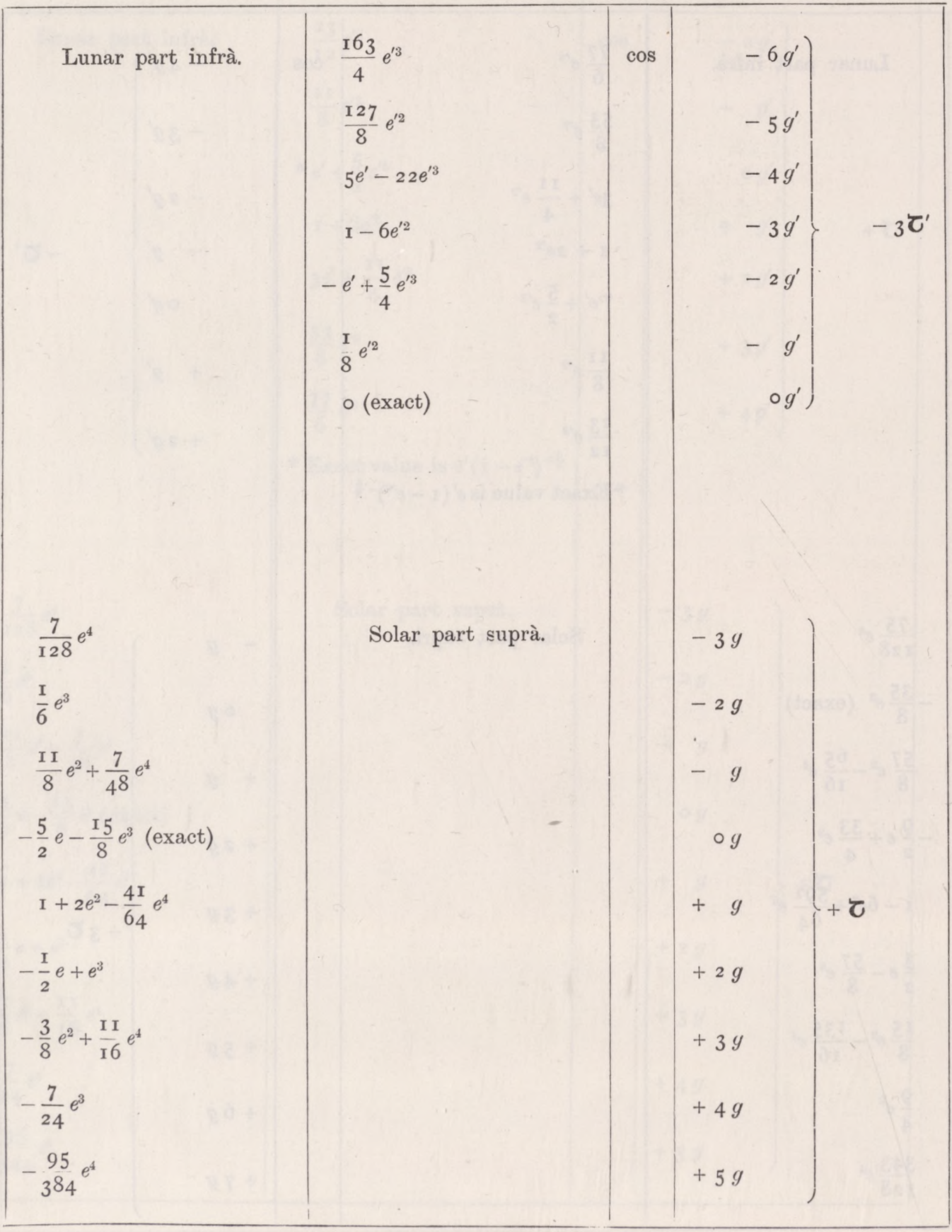


The arrangement of the tables hardly requires any explanation; each line of the upper half of a table is to be read in combination with each line of the lower half of the same table. Thus a term of $\Omega_{2}$ is

$$
m^{\prime} \frac{a^{2}}{a^{\prime 3}}\left(\frac{3}{4}-\frac{3}{2} \eta^{2}+\frac{3}{4} \eta^{4}\right)\left(-\frac{7}{24} e^{3}-\frac{47}{384} e^{5}\right)\left(1-\frac{5}{2} e^{\prime 2}+\frac{13}{16} e^{\prime 4}\right) \cos \left(-g-2 g^{\prime}+2 \boldsymbol{乙}-2 \boldsymbol{乙}^{\prime}\right) .
$$

It is to be observed that in the table for $\Omega_{1}$ (where the arguments depend only on the mean anomalies), the several terms (other than the constant term) occur in pairs of equal terms, having respectively the arguments $i g+i^{\prime} g^{\prime}$ and $-i g-i^{\prime} g^{\prime}$; such equal terms are to be united together, or, what is the same thing, the coefficients must be multiplied by 2 . Thus we have the two terms

$$
\begin{array}{cc}
m^{\prime} \frac{a^{2}}{a^{\prime 3}}\left(\frac{1}{4}-\frac{3}{2} \eta^{2}+\frac{3}{2} \eta^{4}\right)\left(-\frac{9}{160} e^{6}\right)\left(\frac{1773}{256} e^{\prime 5}\right) & \cos \left(-6 g-5 g^{\prime}\right) \\
\text { Ditto } & \cos \left(6 g+5 g^{\prime}\right)
\end{array}
$$

or, what is the same thing, the term is

$$
m^{\prime} \frac{a^{2}}{a^{\prime 3}}\left(\frac{1}{4}-\frac{3}{2} \eta^{2}+\frac{3}{2} \eta^{4}\right)\left(-\frac{9}{160} e^{6}\right)\left(\frac{1773}{256} e^{\prime 5}\right) 2 \cos \left(6 g+5 g^{\prime}\right) .
$$

This is the case even when either $i$ or $i^{\prime}$ vanishes; but, as already noticed, it. is not the case for the constant term where $i$ and $i^{\prime}$ both vanish. There are not any such equal pairs in the tables for $\Omega_{2}$, \&c., where all the arguments contain a part independent of the mean anomalies. The quantity $\tau^{\prime}$ occurs in the upper or solar half of the table; but it is to be recollected that $\tau^{\prime}\left(=\varpi^{\prime}-\Theta\right)$ involves $\Theta$, which is an element of the moon's orbit.

The peculiar form in which the coefficients are exhibited, viz. as the product of a term depending on the inclination, a term depending on the eccentricity of the moon's orbit, and a term depending on the eccentricity of the sun's orbit is not to be considered as a want of completion of the development; it is, on the contrary, an important advantage.

[The Errata in the Tables, noticed Mem. R. Ast. Soc. vol. xxvIII. p. 216, have been corrected. The values of $P^{i}, Q_{c}{ }^{i}, Q_{s}{ }^{i}$, \&c. used in the construction of the Tables ante p. 298 may also be obtained from the Tables of the Developments of Functions in The Theory of Elliptic Motion, 216.]

\section{Addition.}

I deduce from the preceding an expression for the disturbing function in a form similar to and easily comparable with the forms given by Sir J. W. Lubbock in his work On the Theory of the Moon \&c. (London, 1834), pp. 30-35, and by Pontécoulant in the Théorie Analytique du Système du Monde, t. IV. (Paris, 1846), pp. 58-61. The several notations are as follows, viz.: 
Lubbock.

$\xi$,

$\xi^{\prime}$

$\tau$,

$\eta$

$\gamma$,
Pontécoulant.

$\phi$,

$\phi^{\prime}$,

$\xi$,

$\eta$,

$\gamma$,
Suprà.

$g$

$g^{\prime}$

$g-g^{\prime}+\tau-\boldsymbol{乙}^{\prime}$

$g+\tau$

$2 \eta \sqrt{1-\eta^{2}} \div\left(1-2 \eta^{2}\right)$

( $\gamma$, the tangent of the inclination, is employed by the above-named two authors instead of $\eta$, the sine of the semi-inclination, the relation between these two quantities gives to $\gamma^{4}$ or $\eta^{4}$,

$$
\begin{aligned}
& \gamma^{2}=4 \eta^{2}+12 \eta^{4} \\
& \gamma^{4}=16 \eta^{4}
\end{aligned}
$$

and conversely,

$$
\begin{aligned}
& \eta^{2}=\frac{1}{4} \gamma^{2}-\frac{3}{16} \gamma^{4} \\
& \eta^{4}=\frac{1}{16} \gamma^{4}
\end{aligned}
$$

which are useful for replacing one of these quantities by the other). The disturbing function is taken by Lubbock with the contrary sign, and he includes in the beforementioned omitted term depending only on the sun's radius vector, that is, $R$ (Lubbock) $=-m^{\prime} \frac{1}{r^{\prime}}-\Omega ;$ he uses also, in reference to the sun, subscript strokes instead of accents (so that, in referring to his notation, $\xi$, should properly be here used in the place of $\xi^{\prime}$, but this difference is obviously immaterial). Pontécoulant's disturbing function is taken with the same sign as in the present memoir, or we have $R$ (Pontécoulant) $=\Omega$. Lubbock and Pontécoulant give in the principal terms the part involving $m^{\prime} \frac{a^{4}}{a^{\prime 5}}$, which depends on the square of the parallax, and which was disregarded in the preceding development. I have in the sequel inserted these terms from Lubbock's expression. We have, in fact: 
$\Omega=m^{\prime} \frac{a^{2}}{a^{\prime 3}}$ multiplied into

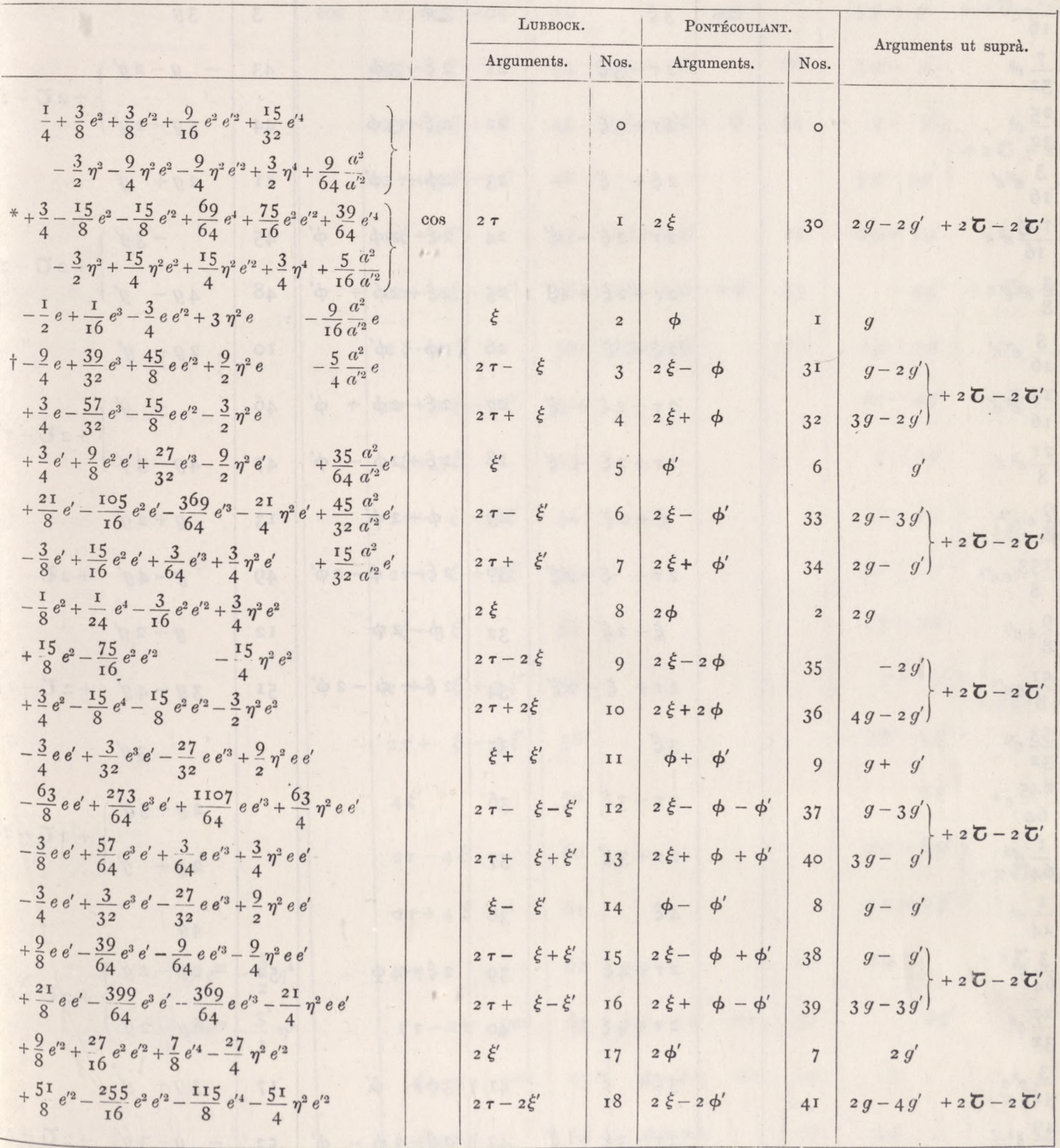

* Variation. + Evection. 
$\Omega($ continued $)=m^{\prime} \frac{a^{2}}{a^{\prime 3}}$ multiplied into

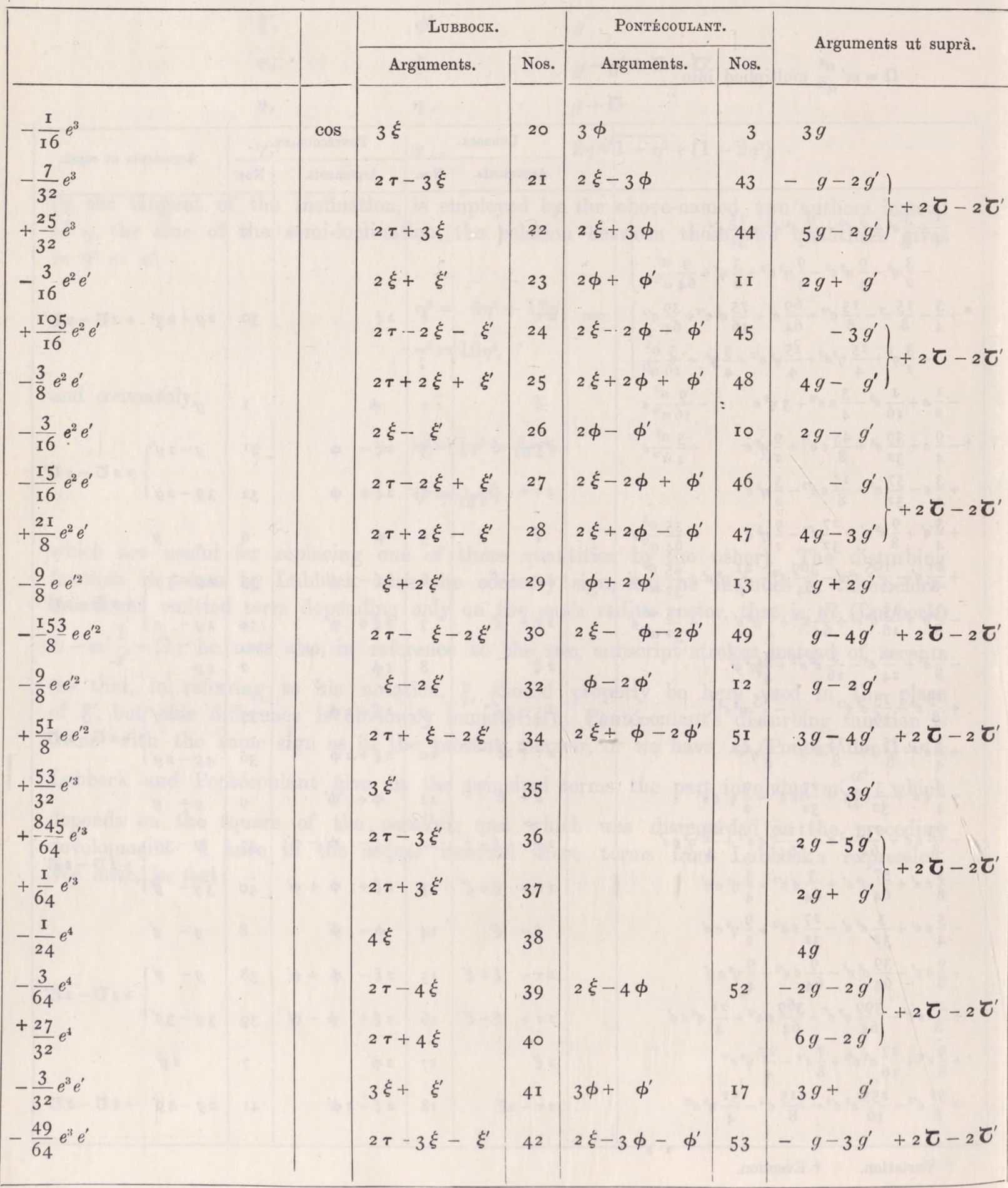


$\Omega($ continued $)=m^{\prime} \frac{a^{2}}{a^{\prime 3}}$ multiplied into

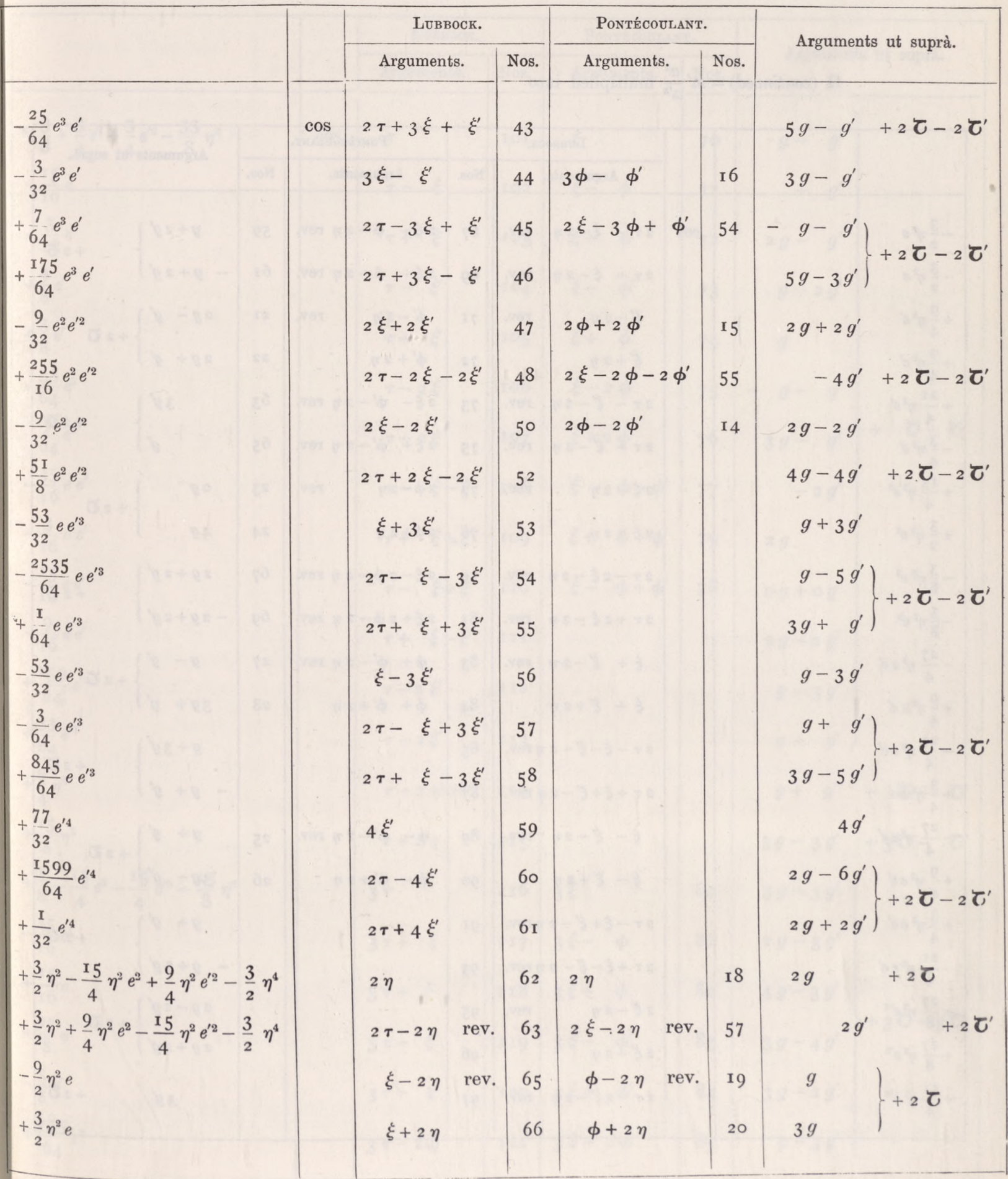


$\Omega($ continued $)=m^{\prime} \frac{a^{2}}{a^{\prime 3}}$ multiplied into

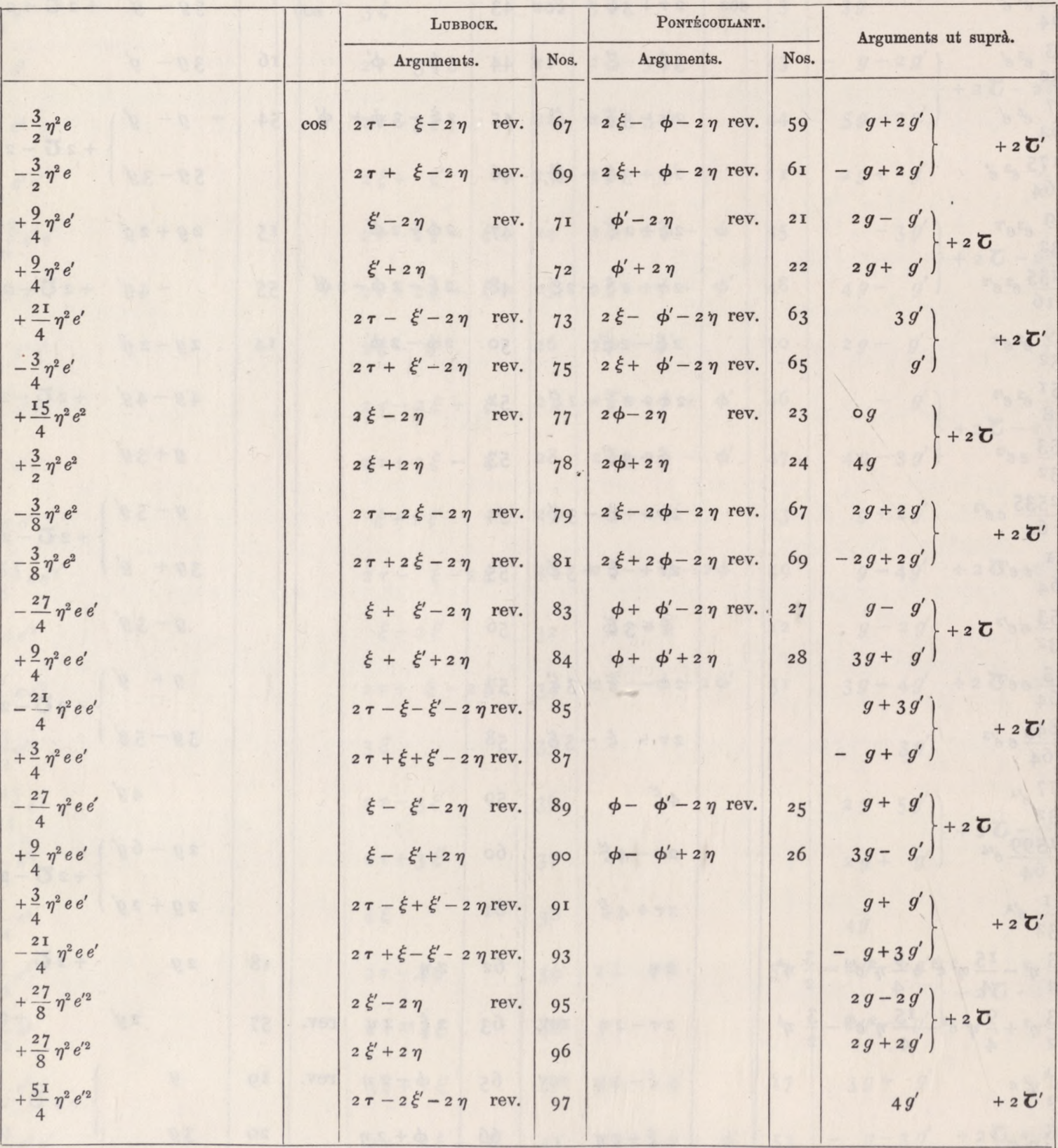


$\Omega($ continued $)=m^{\prime} \frac{a^{3}}{a^{\prime 4}}$ multiplied into

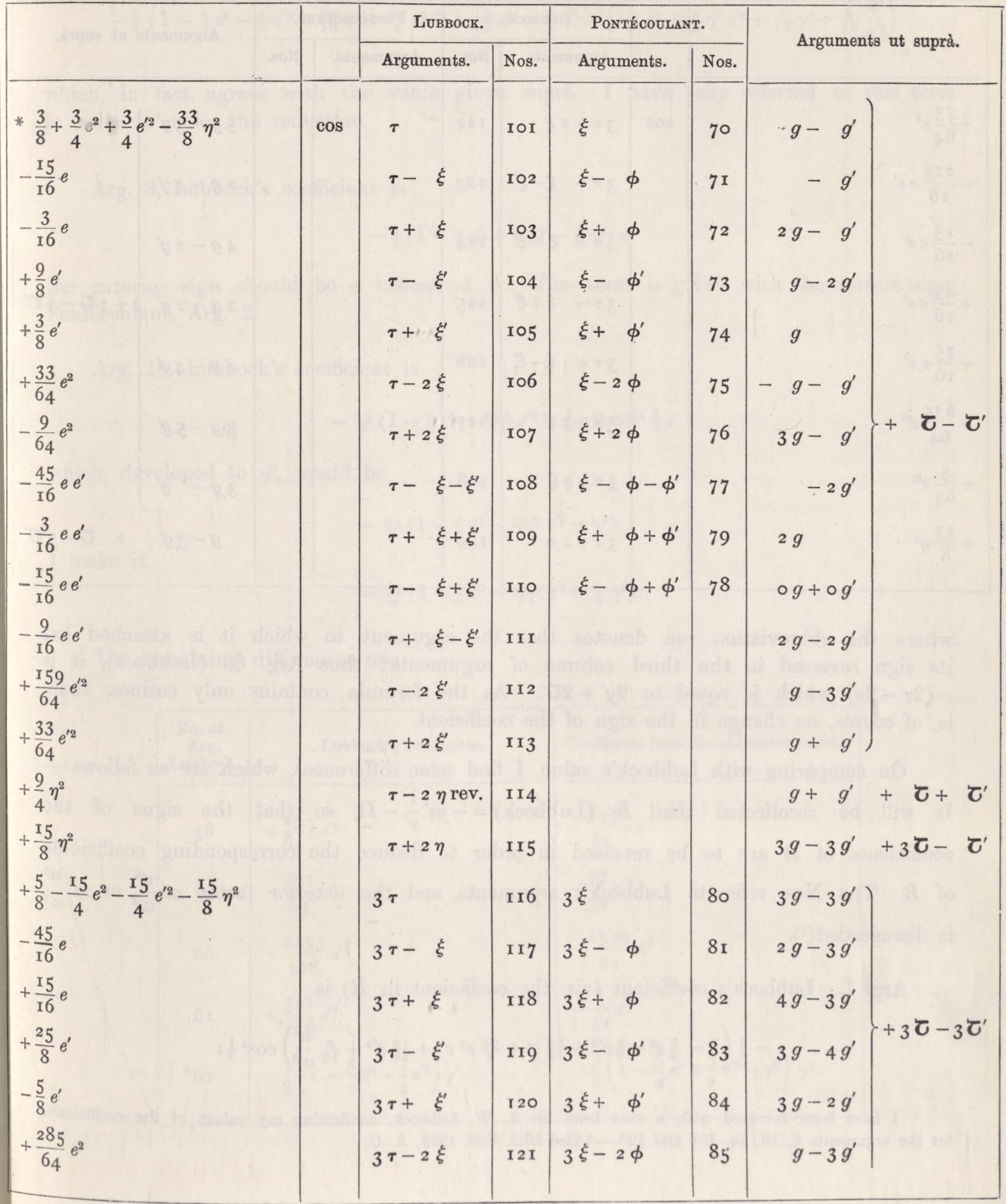

* Parallactic equation. 
$\Omega($ continued $)=m^{\prime} \frac{a^{3}}{a^{\prime 4}}$ multiplied into

\begin{tabular}{|c|c|c|c|c|c|c|c|c|}
\hline & & & Lubвос & & Pontécoul & & \multirow{2}{*}{\multicolumn{2}{|c|}{ Arguments ut suprà. }} \\
\hline & & & Arguments. & Nos. & Arguments. & Nos. & & \\
\hline$+\frac{75}{64} e^{2}$ & $x^{2}=x^{2}$ & $\cos$ & $3 \tau+2 \xi$ & 122 & & & $5 g-3 g^{\prime}$ & \\
\hline$-\frac{225}{16} e e^{\prime}$ & . & & $3^{\tau-} \quad \xi-\xi^{\prime}$ & 123 & & & $2 g-4 g^{\prime}$ & \\
\hline$-\frac{15}{16} e e^{\prime}$ & 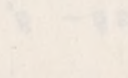 & & $3 \tau+\xi+\xi^{\prime}$ & I 24 & & & $4 g-2 g^{\prime}$ & \\
\hline$+\frac{45}{16} e e^{\prime}$ & & & $3 \tau-\xi+\xi^{\prime}$ & I 25 & & & $2 g-2 g^{\prime}$ & $+3 \boldsymbol{\tau}-3 \boldsymbol{U}^{\prime}$ \\
\hline$+\frac{75}{16} e e^{\prime}$ & & & $3 \tau+\xi-\xi$ & I 26 & & & $4 g-4 g^{\prime}$ & \\
\hline$+\frac{635}{64} e^{\prime 2}$ & & & $3 \tau-2 \xi^{\prime}$ & 127 & & & $3 g-5 g^{\prime}$ & \\
\hline$+\frac{5}{64} e^{\prime 2}$ & & & $3^{\tau+2 \xi^{\prime}}$ & I 28 & te & & $3 g-g^{\prime}$ & \\
\hline$+\frac{15}{8} \eta^{2}$ & & & $3 \tau-\Sigma \eta$ & I 29 & & & $g-3 g^{\prime}$ & $+\boldsymbol{\tau}-3 \boldsymbol{\Xi}^{\prime}$ \\
\hline
\end{tabular}

where the abbreviation rev. denotes that the argument to which it is attached has its sign reversed in the third column of arguments: thus Arg. 63 (Lubbock), it is $-(2 \tau-2 \eta)$ which is equal to $2 g^{\prime}+2 \boldsymbol{\mho}^{\prime}$. As the formula contains only cosines, there is, of course, no change in the sign of the coefficient.

On comparing with Lubbock's value, I find some differences, which are as follows:It will be recollected that $R$ (Lubbock) $=-m^{\prime} \frac{1}{r^{\prime}}-\Omega$, so that the signs of the coefficients of $\Omega$ are to be reversed in order to deduce the corresponding coefficients of $R$. The Nos. refer to Lubbock's arguments, and the exterior factor $m^{\prime} \frac{a^{2}}{a^{\prime 3}}$ or $m^{\prime} \frac{a^{3}}{a^{\prime 4}}$ is disregarded $\left({ }^{1}\right)$.

Arg. 1. Lubbock's coefficient (viz. the coefficient in $R$ ) is

$$
-\frac{3}{4}\left(1-\frac{5}{2} e^{2}-\frac{5}{2} e^{\prime 2}+\frac{23}{16} e^{4}+\frac{25}{4} e^{2} e^{\prime 2}+\frac{13}{16} e^{\prime 4}+\frac{5}{12} \frac{a^{2}}{a^{\prime 2}}\right) \cos ^{4} \frac{1}{2} \iota
$$

1 I have been favoured with a note from Sir J. W. Lubbock, confirming my values of the coefficients for the arguments 8, 18, 58, 101 and 123.-Added 15th Feb. 1859, A. C. 
which, substituting for $\cos ^{4} \frac{1}{2} \iota$ its value $1-\frac{1}{2} \gamma^{2}+\frac{7}{16} \gamma^{4}-\& c$., and developing to the fourth order, gives

$$
-\frac{3}{4}\left(1-\frac{5}{2} e^{2}-\frac{5}{2} e^{\prime 2}+\frac{23}{16} e^{4}+\frac{25}{4} e^{2} e^{\prime 2}+\frac{13}{16} e^{\prime 4}-\frac{1}{2} \gamma^{2}+\frac{5}{4} \gamma^{2} e^{2}+\frac{5}{4} \gamma^{2} e^{t_{2}}+\frac{7}{16} \gamma^{4}+\frac{5}{12} \frac{a^{2}}{a^{\prime 2}}\right)
$$

which, in fact, agrees with the value given suprà. I have only referred to this term in order to make the reduction.

Arg. 8, Lubbock's coefficient is

$$
-\frac{1}{8}\left(1-\frac{1}{3} e^{2}+\frac{3}{2} e^{\prime 2}-\frac{3}{2} \gamma^{2}\right) e^{2}
$$

the exterior sign should be + instead of - . The term is given with the correct sign, Pontécoulant, Arg. 2.

Arg. 18, Lubbock's coefficient is

$$
-\frac{5 \lambda}{8}\left(1-\frac{5}{2} e^{2}-\frac{115}{51} e^{\prime 2}-\frac{1}{2} \gamma^{2}\right) \cos ^{4} \frac{1}{2} \iota
$$

which, developed to $\gamma^{2}$, would be

$$
-\frac{51}{8}\left(1-\frac{5}{2} e^{2}-\frac{115}{51} e^{\prime 2}-\gamma^{2}\right)
$$

I make it

$$
-\frac{51}{8}\left(1-\frac{5}{2} e^{2}-\frac{115}{51} e^{\prime 2}-\frac{1}{2} \gamma^{2}\right)
$$

The remaining differences are

\begin{tabular}{|c|l|l|}
\hline $\begin{array}{c}\text { No. of } \\
\text { Arg. } \\
\text { Lubbock. }\end{array}$ & \multicolumn{1}{|c|}{ Lubbock's coefficient. } & Coefficient from Development suprà. \\
\cline { 1 - 2 } 58 & $+\frac{45}{64} e e^{\prime 3}$ & $-\frac{845}{64} e e^{\prime 3}$ \\
59 & $+\frac{59 \mathrm{I}}{64} e^{\prime 4}$ & $-\frac{77}{32} e^{\prime 4}$ \\
60 & $-\frac{2453}{\mathrm{I} 28} e^{\prime 4}$ & $-\frac{\mathrm{I} 599}{64} e^{\prime 4}$ \\
$6 \mathrm{I}$ & $+\frac{74 \mathrm{I}}{\mathrm{I} 28} e^{\prime 4}$ & $-\frac{\mathrm{I}}{3^{2}} e^{\prime 4}$ \\
${ }^{*} 62$ & $-\frac{3}{8}\left(\mathrm{I}-\frac{5}{2} e^{2}+\frac{3}{2} e^{\prime 2}\right) \gamma^{2}$ & $-\frac{3}{8}\left(\mathrm{I}-\frac{5}{2} e^{2}+\frac{3}{2} e^{\prime 2}-\gamma^{2}\right) \gamma^{2}$ \\
\hline
\end{tabular}


ON THE DEVELOPMENT OF THE DISTURBING FUNCTION \&C.

\begin{tabular}{|c|c|c|}
\hline $\begin{array}{l}\text { No. of } \\
\text { Arg. } \\
\text { Lubbock. }\end{array}$ & Lubbock's coefficient. & Coefficient from Development suprà. \\
\hline *63 & $-\frac{3}{8}\left(\mathrm{I}+\frac{3}{2} e^{2}-\frac{5}{2} e^{\prime 2}+\frac{\mathrm{I}}{8} \gamma^{2}\right)$ & $-\frac{3}{8}\left(\mathrm{I}+\frac{3}{2} e^{2}-\frac{5}{2} e^{\prime 2}-\gamma^{2}\right) \gamma^{2}$ \\
\hline 95 & $-\frac{27}{\mathrm{I} 6} \gamma^{2} e^{\prime 2}$ & $-\frac{27}{3^{2}} \gamma^{2} e^{\prime 2}$ \\
\hline 96 & $-\frac{27}{16} \gamma^{2} e^{\prime 2}$ & $-\frac{27}{3^{2}} \gamma^{2} e^{\prime 2}$ \\
\hline *IOI & $-\frac{3}{8}\left(\mathrm{I}+3 e^{2}+3 e^{\prime 2}-\frac{\mathrm{II}}{4} \gamma^{2}\right.$ & $-\frac{3}{8}\left(\mathrm{I}+2 e^{2}+2 e^{\prime 2}-\frac{\mathrm{II}}{4} \gamma^{2}\right)$ \\
\hline II 5 & $-{ }_{128}^{15} \gamma^{2}$ & $-\frac{15}{3^{2}} \gamma^{2}$ \\
\hline 123 & $-\frac{225}{16} e e^{\prime}$ & $+\frac{225}{16} e e^{\prime}$ \\
\hline
\end{tabular}

The greater part of the discordant terms do not occur in Pontécoulant's development, which is not carried so far, and the only differences which $I$ find in the coefficients of Pontécoulant's $R(=\Omega)$ are, as regards the arguments 18,57, 70, corresponding respectively to Lubbock's arguments $62,63,101$, included in the preceding table, and for which Pontécoulant's coefficients, correcting for the change of sign, correspond with those given by Lubbock. But I see no room for a mistake in the preceding investigation as regards the coefficients of these three terms; the terms in $\eta$ of the coefficients of 62 and 63 are simply the quantity $\left(\frac{3}{2} \eta^{2}-\frac{3}{2} \eta^{4}\right)$, which forms the exterior factor of $\Omega_{3}$ and $\Omega_{4}$ respectively, and which, putting for $\eta^{2}$ and $\eta^{4}$, their values, is equal to $\frac{3}{8}\left(1-\gamma^{2}\right) \gamma^{2}$, and as regards the coefficient of 101 , the portion $1+2 e^{2}+2 e^{\prime 2}$ of this coefficient is obtained by the mere multiplication of the factors $1+2 e^{2}-\&$ c. and $1+2 e^{\prime 2}$ set opposite to $g+\tau$ and $0 g^{\prime}-\boldsymbol{\tau}^{\prime}$ respectively in the table for $\Omega_{6}$.

2, Stone Buildings, W.C., 7th July, 1858. 